\title{
Claudin-11 decreases the invasiveness of bladder cancer cells
}

\author{
NINAAD S. AWSARE ${ }^{1}$, TRACEY A. MARTIN ${ }^{1}$, MARK D. HAYNES ${ }^{1}$, PHILIP N. MATTHEWS ${ }^{2}$ and WEN G. JIANG ${ }^{1}$ \\ ${ }^{1}$ Metastasis and Angiogenesis Research Group, Cardiff University School of Medicine; \\ ${ }^{2}$ Department of Urology, University Hospital of Wales, Cardiff CF14 4XN, UK
}

Received December 2, 2010; Accepted January 17, 2011

DOI: $10.3892 / o r .2011 .1244$

\begin{abstract}
The expression of claudin-11 in benign and malignant bladder tissue and the effect of forced expression of claudin-11 on tight junction function and invasiveness of bladder cancer cells were studied. Claudin-11 expression was tested in bladder cancer cell lines (T24/83, RT 112/84 and EJ138) using reverse transcription-polymerase chain reaction (RT-PCR) and in benign and malignant bladder tissue by quantitative RT-PCR and immunohistochemistry. T24/83 cells were transfected with the pcDNA.1/NT-GFPTOPO vector containing full-length human claudin-11 sequence. Stable-transfected cells overexpressing claudin-11 (T24 ${ }^{\mathrm{Cl}-11 \mathrm{Ex}}$ ), wild-type cells (T24 ${ }^{\mathrm{WT}}$ ) and the empty plasmid control clone (T24 $\left.{ }^{\mathrm{GFP}}\right)$ were compared using transurothelial resistance (TUR), in vitro adhesion, invasion and growth assays. Claudin-11 was strongly expressed in the non-invasive RT112/84 cell line compared to the invasive T24/83 and EJ138 TCC cell lines. Benign bladder tissue demonstrated equal expression of claudin-11 mRNA as carcinoma, but displayed more intense staining than malignant tissue on immunohistochemistry. Forced-expression of claudin-11 in T24/83 cells was confirmed by PCR, immunoprecipitation and by immunofluorescence, which demonstrated increased perinuclear claudin-11 staining. Forced expression of claudin-11 did not affect TUR $(p=0.243)$, but significantly reduced invasion $(\mathrm{p}=0.001)$ while increasing cell matrix adhesion $(\mathrm{p}=0.001)$ and growth rates $(\mathrm{p}=0.001)$. The greater expression of claudin-11 in benign vs. malignant tissue and non-invasive vs. invasive cell lines, and its effect in reducing bladder cancer cell invasiveness suggests that claudin-11 may have a role in preventing cancer
\end{abstract}

Correspondence to: Professor Wen G. Jiang, Metastasis and Angiogenesis Research Group, Cardiff University School of Medicine, Heath Park, Cardiff CF14 4XN, UK

E-mail: jiangw@cf.ac.uk

Abbreviations: CK-19, cytokeratin-19; ECM, extracellular matrix; GFP, green fluorescent protein; MTT, 3-(4, 5-dimethylthiazol-2yl)-2, 5-diphenyl-tetrazolium bromide; PCR, polymerase chain reaction; Q-RT-PCR, quantitative-reverse transcription-PCR; TCC, transitional cell carcinoma; TJ, tight junction; TBS, Tris-buffered saline; TUR, transurothelial resistance; ZO, zona occludens

Key words: urinary bladder neoplasms, tight junctions, membrane proteins, CLDN11 protein, human neoplasm metastasis progression and may serve as a therapeutic target in reducing metastasis.

\section{Introduction}

Bladder cancer is the seventh most commonly diagnosed cancer and the eighth most common cause of cancer-related death in the UK, accounting for 5002 deaths in 2008 (1). In the United States, an estimated 14,680 persons will die of bladder cancer in 2010 (2). Approximately $70 \%$ of newly diagnosed bladder cancers are superficial, low-grade tumors, which are managed endoscopically, with or without intravesical chemotherapy or immunotherapy (3). However, up to $70 \%$ of these patients develop tumour recurrence and $10-15 \%$ progress to muscle-invasive disease requiring radical treatment. Approximately $30 \%$ of patients with muscle-invasive disease have undetectable metastasis at diagnosis (4), which portends a poor prognosis.

Metastasis is a complex process characterised by increased tumour cell proliferation and motility, stromal invasion, angiogenesis, intravasation of cells into the circulation and finally extravasation and establishment of distant metastases. A breakdown in the mechanisms controlling normal cell-cell adhesion is critical in the growth and progression of cancer. In the normal urothelium, tight junctions (TJ) and adherens junctions (AJ) play a major role in maintaining cell-cell adhesion. TJs have a number of diverse functions including the maintenance of transepithelial resistance and the control of paracellular permeability across epithelial surfaces $(5,6)$, the maintenance of cell polarity (7), cell signalling and cytoskeletal regulation (8) and cell growth and differentiation (9). In the mammalian bladder TJs consist of 4-6 interconnecting strands that obliterate the intercellular space (10). The TJs are made up of the transmembrane proteins (including occludin and the claudins) that form the $\mathrm{TJ}$ strands and the peripheral plaque proteins (ZO-1, ZO-2, ZO-3, AF6 and MUPP-1) that bind the transmembrane proteins with the cytoskeleton (11). The claudins define the permeability characteristics of epithelial and endothelial tissues (6).

Although previous reports have described the tissue distribution of claudins and their altered expression in a number of cancers (12), there are few reports on their expression in the urothelium. Claudins $-4,-8$ and -12 were expressed in the umbrella cell TJs of murine epithelium (13), while claudins-3, -4, -5 and -7 were expressed in human urothelium (14). Claudin-4 expression was downregulated in bladder cancer compared 
to normal urothelium and it localised to the cytoplasm rather than the cell membrane in the cancer cells (15). We analyzed the expression of a number of claudins in TCC cell lines and in bladder tissue. Since the expression of claudin-11 was decreased in the invasive cell lines compared to the non-invasive ones, and in TCC tissue as compared to benign urothelium, we proceeded to force express claudin-11 in the invasive T24/83 cell line to determine its effect on cell function.

\section{Materials and methods}

Materials. Three bladder cancer cell lines (the low-grade RT112/84, and the high-grade invasive cell lines T24/83 and EJ138) were obtained from the European Collection of Animal Cell Culture (ECACC, Salisbury, UK) and maintained in Dulbecco's modified Eagle's medium/Ham's F-12 medium supplemented with $10 \%$ heat-inactivated foetal calf serum, benzyl-penicillin and streptomycin. Primers (Invitrogen, Paisley, UK) and anti-claudin-11 and anti-ZO-1 antibodies (Santa Cruz Biotechnology, Santa Cruz, USA) were used in the study.

Immunohistochemical staining of frozen bladder specimens. Snap-frozen bladder tissue samples (cancer and benign background) were obtained from patients undergoing transurethral resection of bladder tumour or radical cystectomy following ethics committee approval and written, informed consent. All biopsies were also histopathologically evaluated to confirm if they were cancer or benign tissue.

We used a previously reported protocol to stain bladder tissue specimens for claudin-11 with anti-claudin-11 rabbit antibody (1:100 dilution) (16,17). The staining intensity in benign and malignant tissue was quantified using Optimas 6.0 software and compared as previously described $(17,18)$.

RNA isolation and RT-PCR. RNA was extracted using Total RNA isolation reagent (ABgene ${ }^{\mathrm{TM}}$, Epsom, Surrey, UK). RT was performed using an Enhanced Avian HS reverse transcriptase-PCR kit (Sigma-Aldrich, Dorset, UK) followed by PCR (REDTaq ${ }^{\mathrm{TM}}$ ReadyMix $^{\mathrm{TM}}$ PCR Reaction mix, SigmaAldrich). Primer sequences are listed in Table I. Cycling conditions were $94^{\circ} \mathrm{C}$ for $5 \mathrm{~min}, 36$ cycles of $94^{\circ} \mathrm{C}$ for $15 \mathrm{sec}$, $55^{\circ} \mathrm{C}$ for $15 \mathrm{sec}, 72^{\circ} \mathrm{C}$ for $15 \mathrm{sec}$, followed by $72^{\circ} \mathrm{C}$ for $7 \mathrm{~min}$. The PCR products were visualized on $2 \%$ agarose gel stained with ethidium bromide (predicted size; claudin-11: $100 \mathrm{bp}$; $\beta$-actin: $580 \mathrm{bp})$.

Quantitative RT-PCR. Q-RT-PCR was performed on bladder tissue samples with the Amplifluor ${ }^{\mathrm{TM}}$ Uniprimer $^{\mathrm{TM}}$ system (Intergen Company, Oxford, UK) using the iCycler IQ system (Bio-Rad Laboratories, Hemel Hempstead, UK) to detect the expression of claudin-11 (results expressed as sample copy number). Use of standard amount (250 ng) of RNA for all samples, and the detection of CK-19 copy number within these samples were used to normalise the samples, based on previous reports from the host laboratory (16-18). The results were expressed as a ratio of the copy number of claudin-11 to CK-19 in benign and cancer tissue. Primer sequences are described in Table I.

Forced overexpression of claudin-11 in T24/83 cells. Expression primers (Cldn11ExF3 + Cldn11ExR1; predicted size 623 bp) were designed using the Beacon Designer software programme (Premier Biosoft International, Palo Alto, CA, USA) to amplify the full coding region of human claudin-11 based on the Homo sapiens claudin-11 (PubMed accession no. NM1001346) mRNA sequence and are described in Table I. Full length claudin-11 was cloned into the pcDNA3.1/NT-GFP-TOPO TA Expression kit (Invitrogen, Paisley, Scotland, UK), the direction and sequence of the insert verified and the plasmid was extracted. The claudin-11 expression cassette and an empty plasmid was transfected into wild-type T24/83 TCC cells $\left(\mathrm{T} 24^{\mathrm{WT}}\right.$ ) using an Easyjet Plus ${ }^{\circledR}$ electroporator to produce the T24 ${ }^{\text {Cldn-11Ex }}$ and T24 ${ }^{\mathrm{GFP}}$ clones respectively, which were incubated in G418 selection medium.

Sodium dodecyl sulfate-polyacrylamide gel electrophoresis and Western blot. The protein concentration in cell lysates was quantified using a DC Protein Assay kit (Bio-Rad $\left.{ }^{\mathrm{TM}}\right)$. Equal amounts of protein were separated by SDS-PAGE, blotted onto nitrocellulose sheets and probed with anti-claudin-11 primary and peroxidise-conjugated secondary antibodies. The protein bands were visualized by a SuperSignal ${ }^{\mathrm{TM}}$ system and the images documented using the UVIprochemi system (UVItec, Cambridge, UK).

Immunoprecipitation. Immunoprecipitation was used to detect differences in expression of claudin-11 in the wild-type and transformed cell lines, since the results of SDS-PAGE Western blotting were equivocal even after using claudin antibodies reacting to different epitopes. Anti-claudin-11 antibody was added to the cell lysates and the antigen-antibody complexes thus formed were mopped up by a mixture of staphylococcal protein $A$ and protein $\mathrm{G}$ beads, which are covalently bound to sepharose or agarose. These immunocomplexes were separated out by precipitation, followed by SDS-PAGE, and then immuno-probed with secondary antibodies and the bands visualised as above.

Immunofluorescent staining of bladder cancer cells. T24 ${ }^{\mathrm{WT}}$, T24 ${ }^{\mathrm{GFP}}$ and T24 ${ }^{\mathrm{Cldn}-11 \mathrm{Exp}}$ cells were grown to confluence in 16-well chamber slides (Lab-Tek ${ }^{\circledR}$, Nalge-Nunc Inc., Naperville,

Table I. PCR primer sequences.

Sense primer

Anti-sense primer

\section{Claudin-11}

CK19

Actin coggtgtggctaagtaca

caggtccgaggttactgac

caggaggttgaaggactaaa actgaacctgaccgtacacacacagggaaccagatg actgaacctgaccgtacacagtttctgccagtgtgtcttc gggatcagttttctttgtca 

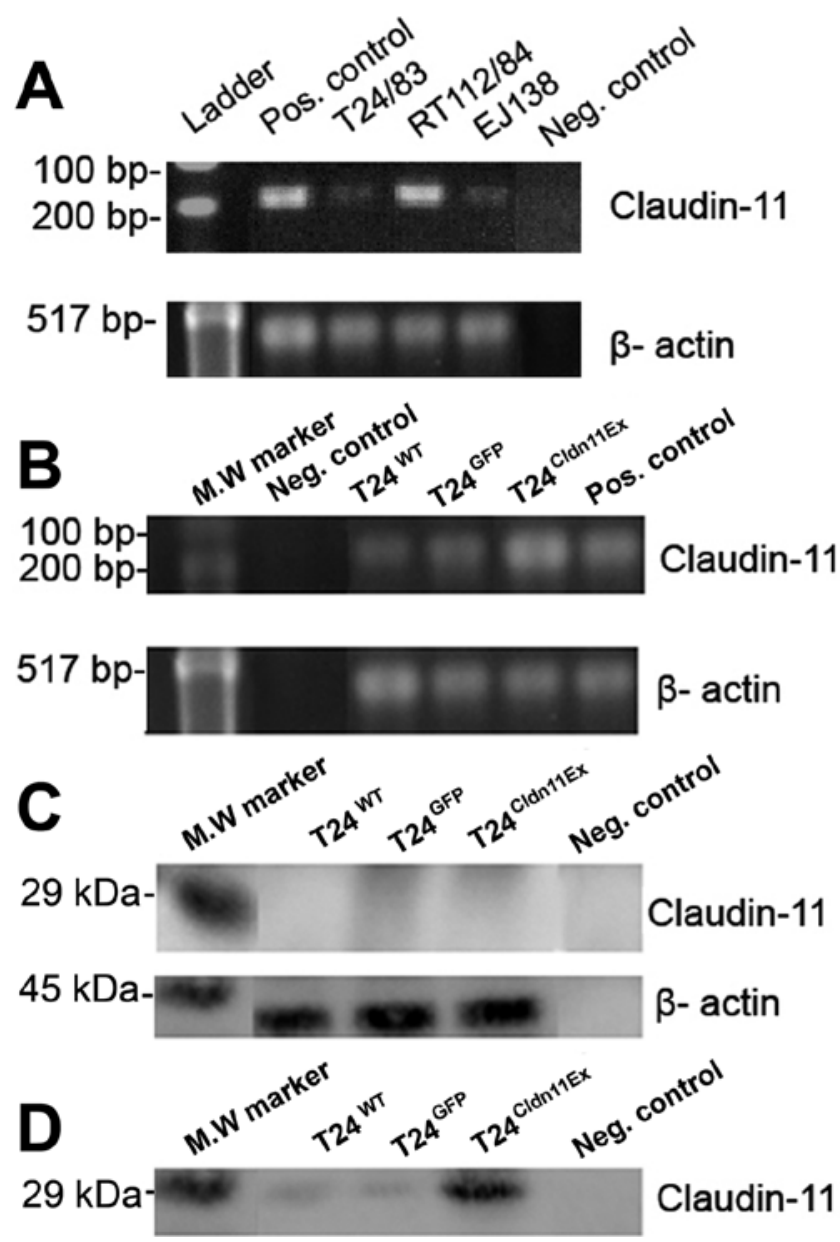

Figure 1. (A) RT-PCR shows claudin-11 expression in T24/83, RT112/84 and EJ138 bladder cancer cell lines. Expression was higher in the non-invasive RT112/84 cell line compared to the invasive (T24/83 and EJ138) cell lines. Pos., positive; Neg., negative. (B) Forced over-expression of claudin-11 in T24/83 cells: RT-PCR demonstrating that claudin-11 mRNA expression was increased in T24Cldn-11Ex compared to T24 ${ }^{\mathrm{WT}}$ and T24 $4^{\mathrm{GFP}}$ cells. (C) Western blot reveals that claudin-11 protein was not detected in any of the three types of cells studied. MW, molecular weight. (D) Immunoprecipitation demonstrating increased expression of claudin-11 protein in T24Cldn-11Ex compared to $\mathrm{T} 24^{\mathrm{WT}}$ and $\mathrm{T} 24^{\mathrm{GFP}}$ cells

USA), fixed in methanol at $-20^{\circ} \mathrm{C}$, washed with TBS, and permeabilised with $0.1 \%$ Triton X-100 for $1 \mathrm{~min}$. Non-specific binding sites were blocked with $10 \%$ horse serum for $60 \mathrm{~min}$. The cells were incubated with anti-claudin-11 or anti-ZO-1 primary antibodies (1:300 dilution), followed by tetramethylrhodamine isothyocyanate (TRITC)-conjugated secondary antibody, mounted using Fluorsave ${ }^{\mathrm{TM}}$ and visualized under a fluorescent microscope.

Transurothelial resistance (TUR). TUR was measured using a previously described method (19). A 24-well plate with $0.4-\mu \mathrm{M}$ pore size cell culture inserts $\left(\right.$ Costar $^{\circledR}$, Corning Inc., NY, USA) was used to grow a confluent layer of each cell type (T24 ${ }^{\mathrm{WT}}, \mathrm{T} 24^{\mathrm{GFP}}, \mathrm{T} 24^{\mathrm{C} 111 \mathrm{Ex}}$ ) using $10^{5}$ cells and the medium was then changed. Resistance was measured using an $\mathrm{EVOM}^{\mathrm{TM}}$ voltohmometer (World Precision Instruments, FL, USA) equipped with a pair of Costar electrodes at baseline ( $0 \mathrm{~min}$ ), 30 min, 1, 2 and $4 \mathrm{~h}$. Results were expressed as a change in
TUR from the value at baseline. Statistics were calculated using repeated measures ANOVA.

In vitro invasion assay. This was performed as previously reported and modified in our laboratory $(16,17$, by adding a total of 10,000 cells to each insert and incubating for $96 \mathrm{~h}$. Cells that had invaded Matrige ${ }^{\mathrm{TM}}$ matrix (BD Biosciences, Bedford, MA, USA) were stained with crystal violet, the dye extracted using 10\% (v/v) acetic acid and absorbances obtained using a multiplate reader $(16,17)$.

Cell matrix adhesion assay. A 96-well tissue culture plate was coated with $1 \mu \mathrm{g} / \mathrm{well}$ of Matrigel, the wells were blocked with 5\% BSA (bovine serum albumin) in sterile BSS (balanced salt solution), and 10,000 of the respective cells were added to each well. After $40 \mathrm{~min}$ of incubation, non-adherent cells were washed off using BSS. Adherent cells were stained with MTT and staining was determined by measuring absorbance on a plate reading spectrophotometer (Bio-Tek ELx 800, Wolf Laboratories, York, UK).

In vitro cell growth assay. This assay was based on a method previously reported by the host laboratory $(16,17)$. Two thousand cells of each type were incubated in a 96-well plate and their growth assessed at days one and five. The cells were stained with crystal violet and absorbance determined using a spectrophotometer. Absorbances represented the cell number, and the percentage change in absorbance at day five compared to day one represented the corresponding change in the number of cells.

Statistical analysis. SPSS ${ }^{\circledR}$ version 16 was used for analysis. Kolmogorov-Smirnov assumptions of normality and homogeneity of variances using the Levene statistic were tested. ANOVA was used to compare the means between groups with Bonferroni post hoc comparisons used to determine the statistical difference between the groups. Differences were considered statistically significant at $\mathrm{p}<0.05$.

\section{Results}

Expression of claudin-11 in T24/83, RT112/84 and EJ 138 bladder cancer cell lines. Claudin-11 was strongly expressed in the non-invasive RT112/84 cell line, while it was only weakly expressed in the invasive T24/83 and EJ138 TCC cell lines (Fig. 1A). Since the expression of claudin-11 in noninvasive and invasive cell lines was not equal, its expression in benign and malignant bladder tissue was investigated to determine any differences in expression and based on these results, we forced-expressed claudin-11 in the invasive T24/83 TCC cell line.

Expression of claudin-11 in benign and malignant bladder tissue

Expression of claudin-11 mRNA in bladder tissue. Eight benign and 17 TCC bladder tissue samples were available for Q-RT-PCR. There was no significant difference $(\mathrm{p}=0.317$, two-tailed t-test) in the claudin-11/CK-19 transcript ratio between benign (mean \pm SEM, 3.81 \pm 0.43 ) and TCC bladder (4.36 \pm 0.31$)$ tissue, implying that there was no significant differ- 
A

A Benign bladder tissue
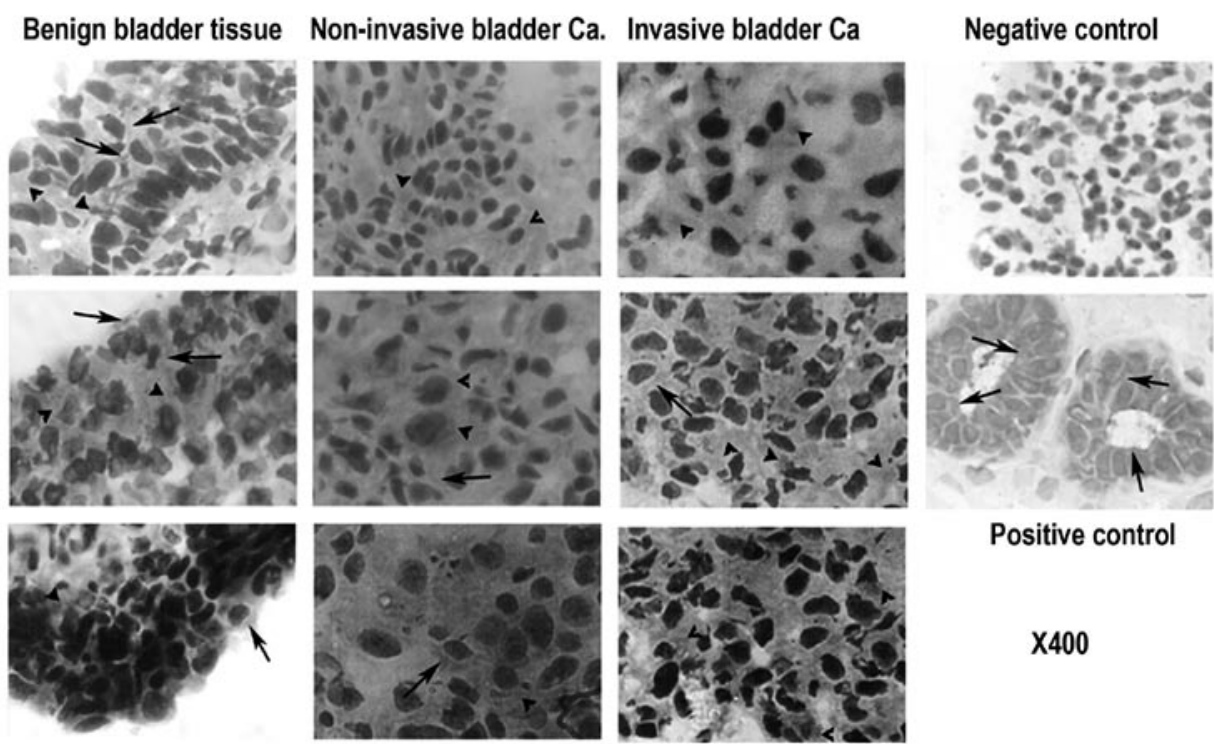

$\mathrm{X} 400$

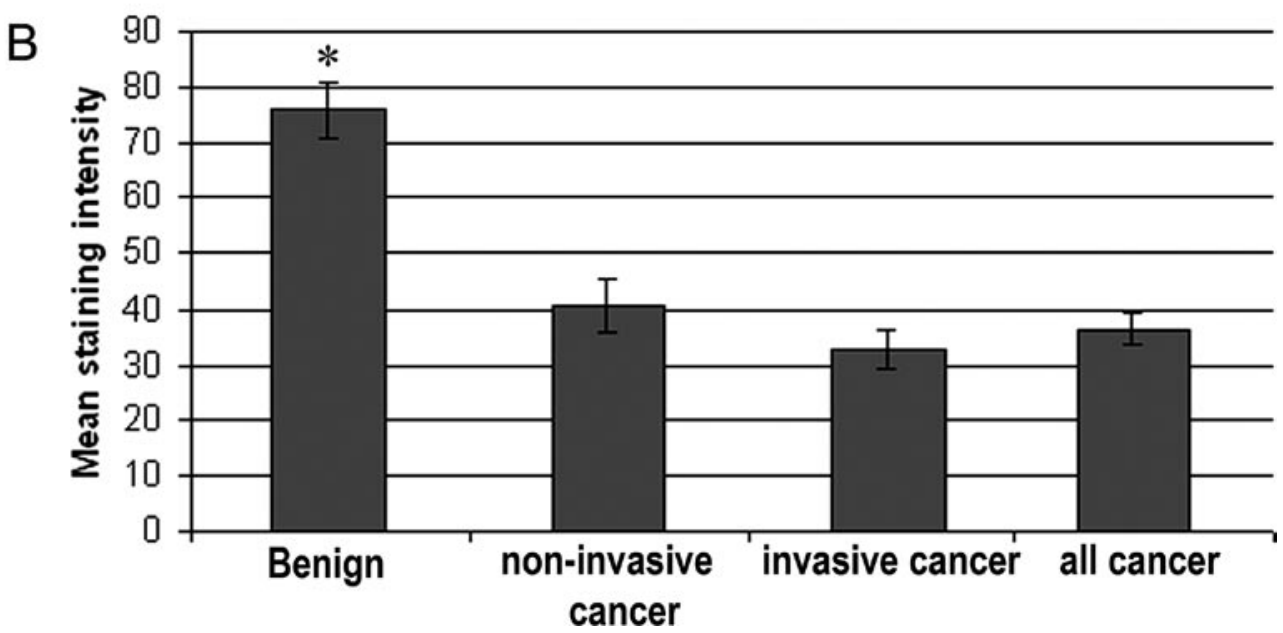

Figure 2. Representative images of claudin-11 immunohistochemical analysis comparing staining patterns and intensity in benign, non-invasive and invasive bladder cancer tissue. (A) There was greater uptake in benign tissue as compared to TCC. Claudin-11 located to the intercellular region and cytoplasm of cells (x400 magnification). (B) Claudin-11 staining intensity was greater in benign bladder tissue, compared to non-invasive, invasive and all cancer tissue. * $<0.05$. Error bars represent SEM.

ence in the expression of claudin-11 mRNA between benign and TCC tissue.

Immunohistochemical expression of claudin-11 protein in bladder tissue. Eight benign and 19 (9 non-invasive and 10 invasive) TCC bladder tissue samples were stained for claudin-11 (Fig. 2A), which demonstrated that claudin-11 located to all layers of the urothelium, but was particularly strongly expressed in the umbrella cell layer. Claudin-11 mainly located to the intercellular region (TJ), but was also demonstrated a cytoplasmic distribution. Quantitative analysis of claudin-11 staining intensity (Fig. 2B) demonstrated that the expression of claudin-11 was significantly greater in benign (mean \pm SEM, 75.9 \pm 5.02 ) compared to all cancer $(36.53 \pm 2.96)$ samples $(\mathrm{p}<0.0001$, two-tailed t-test $)$, and to non-invasive $(40.61 \pm 4.64)$ and invasive $(32.85 \pm 3.68)$ cancer samples $(\mathrm{p}<0.0001$, ANOVA test). Post-hoc analysis using Bonferoni test showed significant difference in staining intensity between benign and non-invasive cancer $(\mathrm{p}<0.001)$ and between benign and invasive cancer tissue $(\mathrm{p}<0.001)$ but no difference between the non-invasive and invasive cancer samples $(p=0.618)$. Thus claudin-11 protein expression was significantly greater in benign bladder tissue compared to TCC.

Forced overexpression of claudin-11 in T24/83 cells. Since the expression of claudin-11 was decreased in TCC bladder tissue compared to benign samples, and in the invasive TCC cell lines compared to the moderately differentiated RT112/84 cell line, we forced expressed claudin-11 in the T24/83 cell line, which was derived from a patient with poorly differentiated recurrent invasive bladder TCC. T24 ${ }^{\text {Cldn-11Ex }}$ cells demonstrated increased expression of claudin-11 (Fig. 1B) mRNA compared to wild-type (T24 ${ }^{\mathrm{WT}}$ ) and empty plasmid containing cells (T24 $\left.{ }^{\mathrm{GFP}}\right)$. Western blotting failed to demonstrate specific claudin-11 bands, but immunoprecipitation using claudin-11 antibody demonstrated increased expression of claudin-11 protein in T24 Cldn-11Ex cells compared to T24 ${ }^{\mathrm{WT}}$ and T24 ${ }^{\mathrm{GFP}}$ (Fig. 1C and D). Immunofluorescence confirmed increased 

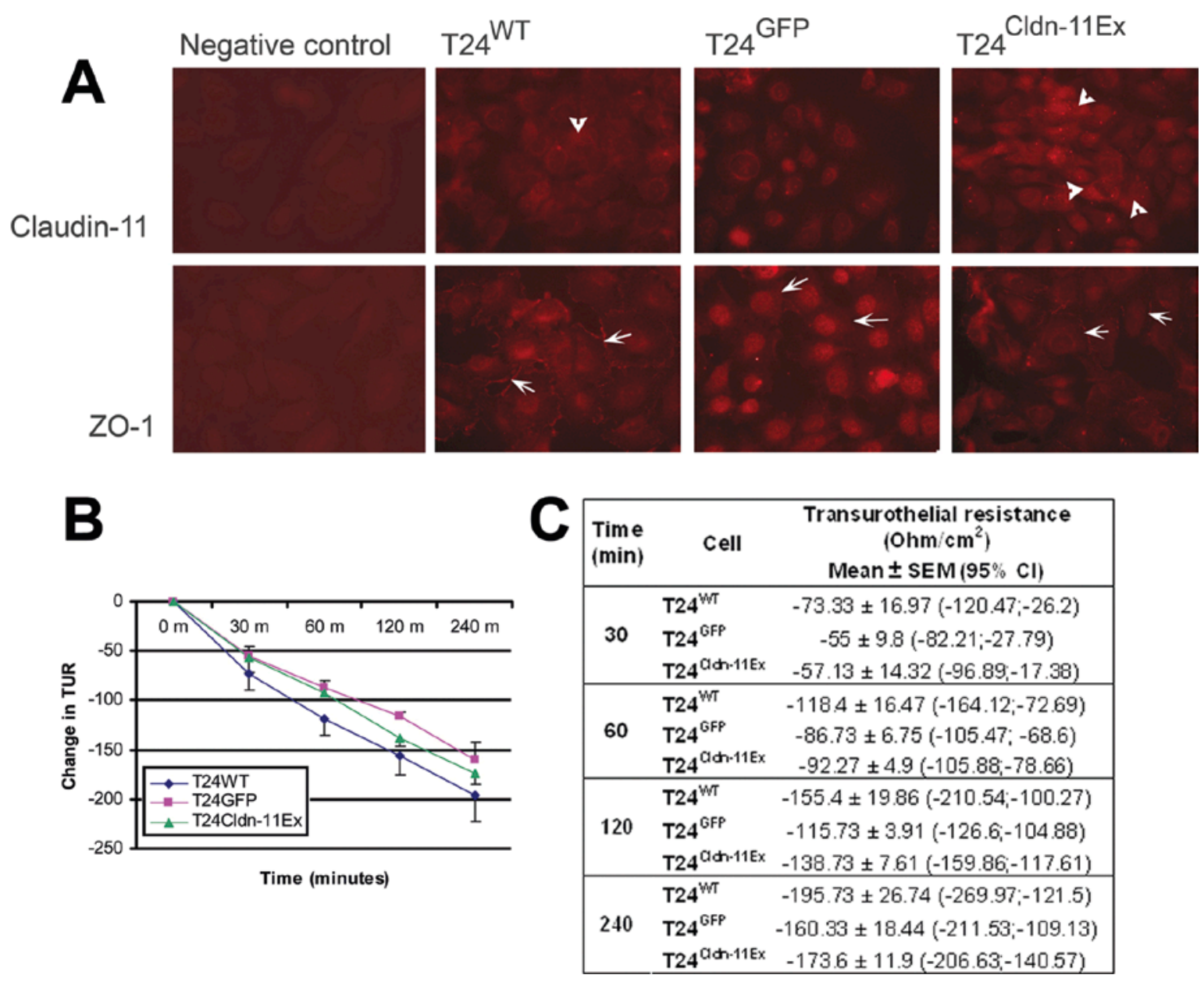

Figure 3. (A) Representative images of immunofluorescence staining for claudin-11 and ZO-1 in T24/83 cells (x40 magnification). There was a stronger signal for claudin-11 in T24 Cldn-11Ex compared to T2 $4^{\mathrm{WT}}$ and T2 $4^{\mathrm{GFP}}$ cells, thus confirming its forced expression. ZO-1 located to tight junctions (white arrows), whereas claudin-11 located to cytoplasm (white arrowheads). (B) Effect of forced-expression of claudin-11 on transurothelial resistance (change from baseline) of T24/83 cells over 240 min. There was no significant difference in the change in TUR in T2 $4^{\text {Cldn-1IEx }}$ cells compared to T2 ${ }^{\mathrm{WT}}$ or T2 $24^{\mathrm{GFP}}$ cells $(\mathrm{p}=0.243$, ANOVA test). Error bars represent SEM. (C) The statistical information of the data set.

expression of claudin-11 protein in the T24 $4^{\text {Cldn-11Ex }}$ cell line compared to controls (Fig. 3A). This mainly located to the cytoplasm and perinuclear region rather than the intercellular region (TJ). Staining for ZO-1 demonstrated intercellular staining (TJ).

Effect of claudin-11 forced expression on transurothelial resistance. Forced expression of claudin-11 or incorporation of GFP plasmid into T24/83 bladder cancer cells had no significant effect ( $p=0.243$, repeated measures ANOVA) on TUR, when measured over $240 \mathrm{~min}$. Fig. $3 \mathrm{~B}$ shows the mean change in TUR from baseline over $240 \mathrm{~min}$ for the T24 cell line.

Effect of claudin-11 forced expression on cell growth. Forced expression of claudin-11 (T24 ${ }^{\mathrm{Cldn}-11 \mathrm{Ex}}$ ) significantly increased ( $\mathrm{p}=0.001$, ANOVA, Fig. 4A and B) the growth of T24/83 bladder cancer cells compared to the T24 ${ }^{\mathrm{WT}}$ and T24 ${ }^{\mathrm{GFP}}$ cells at day 5 . There was however, no difference between T24 ${ }^{\mathrm{WT}}$ and T24 ${ }^{\mathrm{GFP}}$ cells $(\mathrm{p}=1.000)$. Fig. 4B shows the percentage change in absorbance (cell numbers) after 5 days in the T24 cell line.
Effect of claudin-11 forced expression on Matrigel invasion. The technique of in vitro cell matrix invasion involves staining the cells invading through the cell matrix following $96 \mathrm{~h}$ of incubation, with crystal violet and measuring the absorbance of the dye, which is proportional to the number of cells invading through Matrigel. There was a significant decrease ( $p=0.001$, ANOVA, Fig. 4C) in the invasiveness of T24 Cldn-11Ex cells (mean \pm SEM, 1.54 \pm 0.19 ) compared to $\mathrm{T} 24^{\mathrm{WT}}(2.55 \pm 0.33)$ and $\mathrm{T} 24^{\mathrm{GFP}}(2.84 \pm 0.31)$. There was however, no difference between $\mathrm{T} 24^{\mathrm{WT}}$ and $\mathrm{T} 24^{\mathrm{GFP}}$ cells $(\mathrm{p}=1.000)$.

Any change in invasion (cell count) between the control and genetically manipulated groups could either be due to the effect of genetic manipulation on cell growth or on its invasive capacity per se. Since the growth assay revealed that forced expression of claudin-11 (T24 ${ }^{\mathrm{Cldn}-11 \mathrm{Ex}}$ ) significantly increased their growth rate compared to controls, the significant reduction in invasiveness elicited by forced-expression of claudin-11 in T24/83 cells compared to controls may actually underestimate the true effects. 

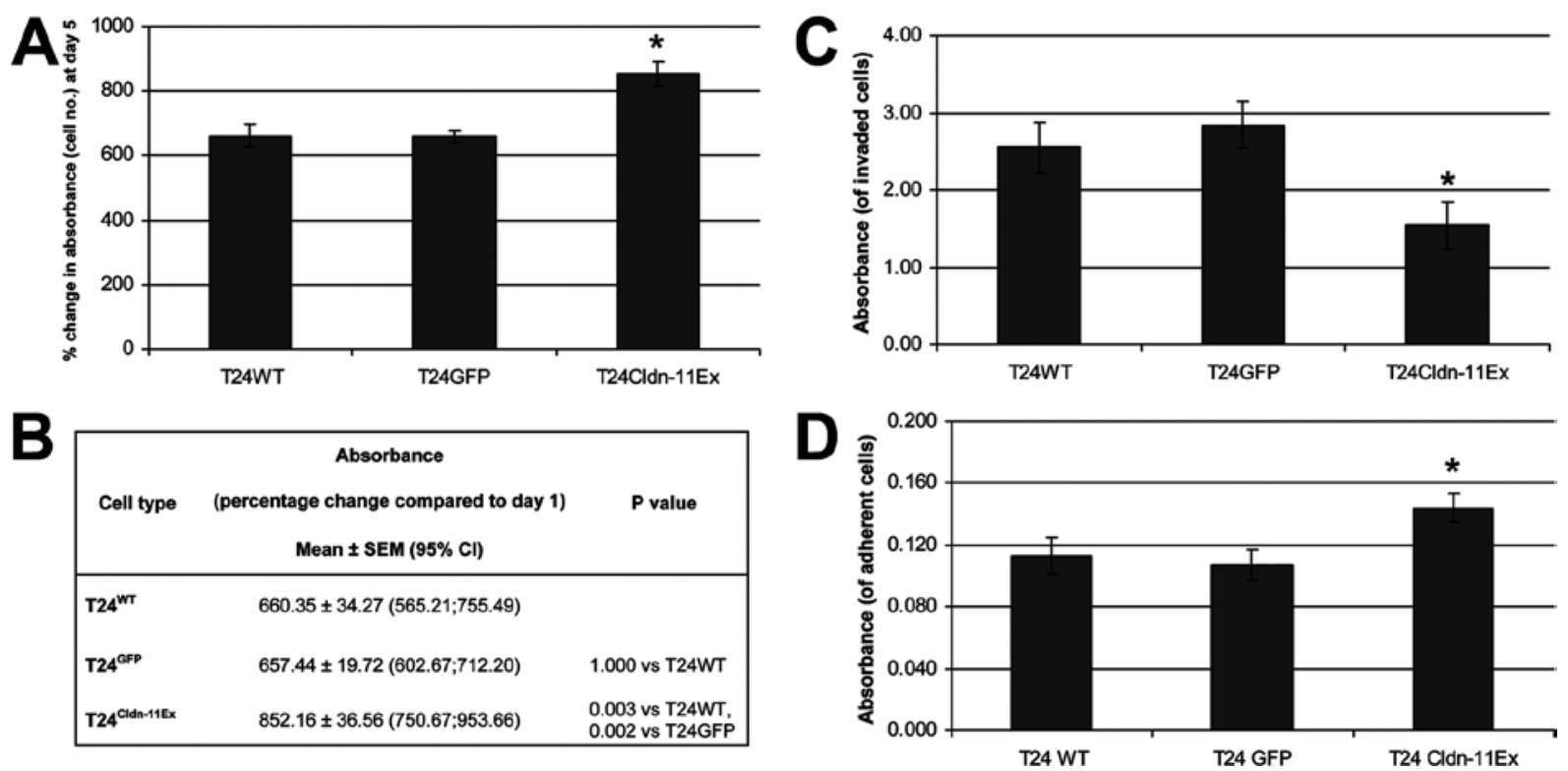

Figure 4. Effect of forced-expression of claudin-11 on T24/83 cells. (A and B) Day 5 change in absorbance, representing percentage change in cell number compared to day 1 . Claudin-11 forced-expression (T24 Cldn-11Ex) significantly increased cell growth compared to T24WT and T24 ${ }^{\mathrm{GFP}}$ cells. " $\mathrm{p}<0.05$. (C) Invasiveness of T24 Cldn-1IEx was significantly reduced compared to T24 ${ }^{\mathrm{WT}}$ and T24 GFP cells. (D) Cell matrix adhesion of T24 ${ }^{\text {Cldn-1IEx }}$ was significantly increased compared to $\mathrm{T} 24^{\mathrm{WT}}$ and T24 ${ }^{\mathrm{GFP}}$ cells. Error bars represent SEM.

Effect of claudin-11 forced expression on cell matrix adhesion. There was a significant increase ( $\mathrm{p}=0.001$, ANOVA, Fig. 4D) in the adhesion of T24 Cldn-11Ex cells (mean \pm SEM, $0.144 \pm 0.009$ ) to Matrigel compared to T24 ${ }^{\mathrm{WT}}(0.113 \pm 0.012)$ and T24 ${ }^{\mathrm{GFP}}$ $(0.107 \pm 0.010)$. There was however, no difference between $\mathrm{T} 24^{\mathrm{WT}}$ and $\mathrm{T} 24^{\mathrm{GFP}}$ cells $(\mathrm{p}=1.000)$.

\section{Discussion}

The TJ of the umbrella cell layer of the urothelium create the paracellular barrier protecting the underlying detrusor against exposure from urinary toxins, with the claudins thought to play a central role in the maintenance of this barrier (6). Disruptions of TJs with loss of TJ proteins including claudins are thought to reduce cell-cell adhesion and lower the diffusion barrier resulting in an abnormal influx of growth factors and nutrients, which offers a selective advantage to developing tumour cells (20). Altered expression of various claudins has been reported in several cancer types (12) with an inverse correlation reported with histological grade (21) and invasiveness (22). Conversely, expression of claudin-3 and -4 positively correlates with invasiveness of ovarian cancer cells (23). Claudin-11 has been reported to be expressed in a number of tissues including the nephron, oligodendrocytes, Sertoli cells and basal cells of the inner ear (24-26) but not previously noted in the urothelium. In the present study, claudin-11 expression was decreased in invasive compared to non-invasive TCC cell lines. The expression of claudin-11 protein was decreased in TCC tissue compared to benign urothelium, although the expression of claudin-11 mRNA was equal in both. This may be due to post-transcriptional and post-translational mechanisms, including histone modification, as has been noted in breast cancer cells where the amount of claudin-1 protein but not mRNA increases as the number of cell contact sites increase (12). In the present study, benign and non-invasive bladder tissue samples showed better formed TJs compared to the invasive samples, possibly explaining the greater degree of claudin-11 expression in the former.

Although Western blotting could not demonstrate forcedexpression of claudin-11 in T24/83, this was confirmed by the more sensitive immunoprecipitation technique. Immunofluorescence studies revealed that claudin-11 located to the peri-nuclear rather than the inter-cellular regions (where ZO-1 signal was detected), suggesting that claudin-11 may not behave like a classic TJ protein in TCC cells. Claudin-4 expression was decreased and reported to delocalise to the cytoplasm in human bladder tumours and the bladder tumour cell line HT-1376 (15). Similarly, claudin-1 was noted along the entire lateral membrane as well as in the cytoplasm of breast cancer cells in tissues (27).

Forced-expression of claudin-11 did not affect TUR of T24/83 cells. This finding is in agreement with the results of immunofluorescent staining suggesting that claudin-11 introduced via plasmid into T24/83 cells was not incorporated into TJs, and hence possibly did not affect TUR. On the other hand, claudin-11 decreased invasion of T24/83 cells through the basement membrane, while increasing their adhesiveness and growth rate. Similarly, forced-expression of claudin-16 in the MDA-MB-231 breast cancer cell line increased their adhesiveness while decreasing invasiveness (28). Although the exact mechanism is unknown, other TJ proteins such as claudin- 3 and -4 increase motility and invasion in ovarian surface epithelial cells by activating matrix metalloproteinase-2 (23), while ZO-1 regulates cell proliferation (9). The results of the functional assays suggest a role for claudin-11 in maintaining tissue barriers, in accord with other reports. Thus, claudin-11 null mice demonstrated hind limb weakness with slowed nerve conduction, impaired spermatogenesis and hearing loss as a result of impaired tissue barrier function $(24,25)$. 
This study is limited by the use of a small number of bladder tissue samples. Further studies must be performed using a large number of samples to confirm the expression of claudin-11 by Western blotting and immunohistochemistry. The suppression of the invasive phenotype by claudin-11 noted in this study needs to be confirmed in vivo and the signalling pathways involved are yet to be identified.

This study demonstrates the role of claudin-11 in bladder cancer cells. Claudin-11 expression could potentially be used as a biomarker to differentiate between non-invasive and aggressive bladder cancer. In vivo efficacy and safety studies must be performed to explore if claudin-11 gene therapy could be used to modify the course of the disease since this therapy can be delivered urethrally directly into the bladder.

\section{Acknowledgements}

Mr. Gareth Watkins, Metastasis and Angiogenesis Research Group, Cardiff University, UK for his advice and technical help in performing the immunohistochemical staining of bladder tissue.

\section{References}

1. Bladder Cancer Statistics-Key Facts. Cancer Research UK. Available at http://info.cancerresearchuk.org/cancerstats/types/ bladder/?a=5441. Accessed August 1, 2010.

2. Cancer of the Urinary Bladder. SEER Stat Fact Sheets: Urinary Bladder. National Cancer Institute. Available at http://seer. cancer.gov/statfacts/html/urinb.html. Accessed August 1, 2010.

3. Bladder Cancer. Bladder cancer statistics and outlook. Cancer Research UK. Available at http://www.cancerhelp.org.uk/type/ bladder-cancer/treatment/bladder-cancer-statistics-and-outlook\#stage. Accessed August 1, 2010.

4. Stenzl A, Cowan N C, De Santis M, et al: The updated EAU guidelines on muscle-invasive and metastatic bladder cancer. Eur Urol 55: 815-825, 2009.

5. Claude P: Morphological factors influencing transepithelial permeability: a model for the resistance of the zonula occludens J Membr Biol 39: 219-232, 1978.

6. Colegio OR, Van Itallie C M, McCrea HJ, et al: Claudins create charge-selective channels in the paracellular pathway between epithelial cells. Am J Physiol Cell Physiol 283: C142-C147, 2002.

7. Kohler K and Zahraoui A: Tight junction: a co-ordinator of cell signalling and membrane trafficking. Biol Cell 97: 659-665, 2005.

8. Matter K and Balda M S: Signalling to and from tight junctions. Nat Rev Mol Cell Biol 4: 225-236, 2003.

9. Balda MS, Garrett MD and Matter K: The ZO-1-associated Y-box factor ZONAB regulates epithelial cell proliferation and cell density. J Cell Biol 160: 423-432, 2003.
10. Peter S: The junctional connections between the cells of the urinary bladder in the rat. Cell Tissue Res 187: 439-448, 1978.

11. Martin TA and Jiang WG: Tight junctions and their role in cancer metastasis. Histol Histopathol 16: 1183-1195, 2001.

12. Swisshelm K, Macek R and Kubbies M: Role of claudins in tumorigenesis. Adv Drug Deliv Rev 57: 919-928, 2005.

13. Acharya P, Beckel J, Ruiz WG, et al: Distribution of the tight junction proteins ZO-1, occludin, and claudin-4, -8 , and -12 in bladder epithelium. Am J Physiol Renal Physiol 287: F305-F318, 2004.

14. Varley CL, Garthwaite MA, Cross W, et al: PPARgammaregulated tight junction development during human urothelial cytodifferentiation. J Cell Physiol 208: 407-417, 2006.

15. Boireau S, Buchert M, Samuel MS, et al: DNA-methylationdependent alterations of claudin- 4 expression in human bladder carcinoma. Carcinogenesis 28: 246-258, 2007.

16. Jiang WG, Martin TA, Lewis-Russell JM, et al: Eplin-alpha expression in human breast cancer, the impact on cellular migration and clinical outcome. Mol Cancer 7: 71, 2008.

17. Jiang WG, Davies G, Martin TA, et al: Targeting matrilysin and its impact on tumor growth in vivo: the potential implications in breast cancer therapy. Clin Cancer Res 11: 6012-6019, 2005.

18. Parr C, Watkins G, Boulton M, et al: Placenta growth factor is over-expressed and has prognostic value in human breast cancer. Eur J Cancer 41: 2819-2827, 2005.

19. Ye L, Martin TA, Parr C, et al: Biphasic effects of 17-beta-estradiol on expression of occludin and transendothelial resistance and paracellular permeability in human vascular endothelial cells. J Cell Physiol 196: 362-369, 2003.

20. Mullin JM: Potential interplay between luminal growth factors and increased tight junction permeability in epithelial carcinogenesis. J Exp Zool 279: 484-489, 1997.

21. Kominsky SL, Argani P, Korz D, et al: Loss of the tight junction protein claudin-7 correlates with histological grade in both ductal carcinoma in situ and invasive ductal carcinoma of the breast. Oncogene 22: 2021-2033, 2003.

22. Michl P, Barth C, Buchholz M, et al: Claudin-4 expression decreases invasiveness and metastatic potential of pancreatic cancer. Cancer Res 63: 6265-6271, 2003.

23. Agarwal R, D'Souza T and Morin PJ: Claudin-3 and claudin-4 expression in ovarian epithelial cells enhances invasion and is associated with increased matrix metalloproteinase-2 activity. Cancer Res 65: 7378, 2005.

24. Gow A, Davies C, Southwood CM, et al: Deafness in claudin 11-null mice reveals the critical contribution of basal cell tight junctions to stria vascularis function. J Neurosci 24: 7051-7062, 2004.

25. Gow A, Southwood CM, Li JS, et al: CNS myelin and sertoli cell tight junction strands are absent in Osp/claudin-11 null mice. Cell 99: 649-659, 1999.

26. Kiuchi-Saishin Y, Gotoh S, Furuse M, et al: Differential expression patterns of claudins, tight junction membrane proteins, in mouse nephron segments. J Am Soc Nephrol 13: 875-886, 2002.

27. Tokes AM, Kulka J, Paku S, et al: Claudin-1, -3 and -4 proteins and mRNA expression in benign and malignant breast lesions: a research study. Breast Cancer Res 7: R296-R305, 2005.

28. Martin TA, Harrison GM, Watkins G, et al: Claudin-16 reduces the aggressive behavior of human breast cancer cells. J Cell Biochem 105: 41-52, 2008. 